\title{
The M 31-WC6 star MLA 1159 and its ionized nebula BA1-642
}

\author{
Gaghik H. Tovmassian ${ }^{1}$, Jochen Greiner ${ }^{2}$, Stefanie Komossa ${ }^{3}$, Margarita \\ Rosado $^{1}$, and Anabel Arrieta ${ }^{1}$ \\ ${ }^{1}$ Instituto de Astronomía, UNAM, B.C., México \\ ${ }^{2}$ Astrophysical Institute Potsdam, Potsdam, Germany \\ ${ }^{3}$ Max-Planck-Institute for extraterrestrial Physics, Garching, Germany
}

\begin{abstract}
Aimed at identifying supersoft X-ray sources in ionization nebulae, we have searched for extended optical emission within the X-ray error boxes of the 15 supersoft sources in M 31 (Greiner et al. 1996). One optical nebula was found, of which we obtained spectroscopy, narrow-band imaging and FPinterferometry, to determine its relation to the positionally correlated supersoft X-ray source RX J0045.5+4206. M 31 has been surveyed several times with different goals. Baade \& Arp (1964) compiled a list of 688 emission nebula in M 31 . One of these nebulae, BA1-642 (PAV 78915 in the catalog of Pellet et al. 1978), was found by cross-correlation with supersoft X-ray sources. The central object MLA 1159 of this nebula has been classified WR (Meyssonier et al. 1993). Here we confirm this new WR star and discuss the star as well the ring-like nebula around it. We did direct imaging of RX J0045.4+4206 with narrow-band nebular filters, spectroscopy of the central star and surrounding nebulae, and we observed the nebula with the FP-interferometer PUMA. All observations were carried out at $O A N$ SPM, México.
\end{abstract}

\section{The central star MLA 1159}

The spectrum of MLA 1159 shows strong and broad emission lines at $4650 \AA$ and $5805 \AA$ interpreted as CIII and CIV and suggesting a WC star, which confirms the WR candidacy proposed by Meyssonier et al. (1993). Classification-line ratios (Smith 1968; van der Hucht et al. 1981; Crowther et al. 1998) classify MLA 1159 as WC6. With $V=19.2$ and assuming a distance of $650 \mathrm{kpc}$, only galactic foreground absorption and no intrinsic M 31 extinction, we find $A_{V}=0.3$ and $M_{V}=-5.7$, two magnitudes brighter than Galactic WC6 stars (van der Hucht et al. 1988).

\section{The nebula BA1-642 (PAV 78915)}

The Wolf-Rayet star MLA 1159 is located within a nearly circular nebula. The SE rim of the nebula is pronounced, particularly in $\mathrm{H} \alpha$. The shape and the size of the nebula is similar at different wavelenghts (HeII, $\mathrm{H} \beta$, [OIII], $\mathrm{H} \alpha$, [NII] and [SII]). The nebula shows strong Balmer emission lines, [OII] and [SII] but weak [OIII]. We note that the emission lines are all blue-shifted by $3-4 \AA$, consistent with the systemic velocity of M 31. We have measured the size of the inner and the outer ring of the nebulae based on the pronounced, bright SE rim visible in the $\mathrm{H} \alpha$ image to $R_{\text {in }} \simeq 11^{\prime \prime}$ and $R_{\text {out }} \simeq 14^{\prime \prime}$. At the distance of M 31 
this corresponds to radii of 34 and $50 \mathrm{pc}$, respectively. Lack of filamentary [OIII] structures together with diffuse [OIII] emission and an identical extent of the nebula at different emission lines, suggests that the nebula is not shock-excited but rather photo-ionized, consistent with the velocity range covered by the nebular emission. With [OIII] $5007 / \mathrm{H} \beta=0.45 \pm 0.08$ as criterion, the excitation class is estimated 0.5 in the system of Feast (1968) and Webster (1975). This and the lack of nebular HeII emission lines indicate that BA1-642 is a low-excitation nebula. The $\mathrm{H} \beta$ luminosity is a few percent of the luminosity of the WR star, and thus can be produced by the irradiation of the central WR star.

\section{Photo-ionization modelling}

We have performed photo-ionization calculations with CLOUDY (Ferland 1993). Our model with ionization parameter $\log U=-3.2$ and $T_{\mathrm{bb}}=55000 \mathrm{~K}$ best fits the observed line ratios. Given that both [NII] and [SII] are correctly predicted by this model, we do not find evidence for a deviation of the $\mathrm{N}$ abundance from the solar value. Under the assumptions made, i.e., dust properties and ISM metal abundances, the observed emission lines are better matched for a dustfree environment (or selectively depleted $S$ abundance). For the best fit, we derive a mean nebular temperature of $T \simeq 5 \times 10^{3} \mathrm{~K}$.

Given the parameters derived above for the luminosity of the WC6 star and the size, density and emission-line strength of the nebula, it seems reasonable to assume that both are physically connected. Because of our initial goal of identifying a supersoft X-ray source, we mention here that the X-ray source RX J0045.5+4206 is very likely not related to either the WC6 star or the nebula. If RX J0045.5+4206 would be within the nebula, it should be the dominant ionization source and produce strong [OIII] emission, e.g., [OIII]/H $\beta \simeq 10-20$, which is not observed. With a bolometric (BB-approximation) luminosity of RX J0045.5+4206 of the order of $10^{37} \mathrm{erg} \mathrm{s}^{-1}$ the X-ray emission is also very unlikely produced by the WC6 star, where Galactic WC6 stars typically have $\mathrm{X}$-ray luminosities of a few times $10^{32} \mathrm{erg} \mathrm{s}^{-1}$.

\section{References}

Baade, W., Arp, H. 1964, ApJ 139, 1027

Crowther, P.A., De Marco, O., Barlow, M.J. 1998, MNRAS 296, 367

Feast, M.W. 1968, MNRAS 140, 345

Ferland, G.J. 1993, Univ. of Kentucky Internal Report

Greiner, J., Supper, R., Magnier, E.A. 1996, Lecture Notes in Phys. 472, 75

van der Hucht, K.A., Conti, P.S., Lundström, I., Stenholm, B. 1981, SSR 28, 227

van der Hucht, K.A., Hidayat, B., Admiranto, A.G., Supelli, K.R., Doom, C. 1988, A\&A 199, 217

Meyssonier, N., Lequeux, J., Azzopardi, M. 1993, A\&AS 102, 251

Osterbrock D.E. 1989, Astrophysics of Gaseous Nebulae and Active Galactic Nuclei (Mill Valley: Univ. Sci. Books)

Pellet, A., Astier, N., Viale, A., Courtes, G., Maucherat, A., Monnet, G., Simien, F. 1978, A\&AS 31, 439

Smith, L.F. 1968, MNRAS 138, 109

Webster, B.L. 1975, MNRAS 173, 437 